\title{
Screening Mammography for Average-Risk Women: The Controversy and NCCN's Position
}

\author{
Mark A. Helvie, MD, ${ }^{a}$ and Therese B. Bevers, MD $^{\mathrm{b}}$
}

\section{Abstract}

Breast cancer remains the most common nonskin cancer among women and a leading cause of morbidity and mortality. Early detection through screening and advances in treatment have contributed to a $39 \%$ mortality reduction in the United States since 1990 . The NCCN Guidelines for Breast Cancer Screening and Diagnosis recommend annual mammographic screening for average-risk women beginning at age 40 years. Mammographic screening and subsequent treatment reduces breast cancer mortality based on a wide range of studies. This article highlights NCCN's position on screening mammography and the screening controversy.

J Natl Compr Canc Netw 2018;16(11):1398-1404 doi: 10.6004/jnccn.2018.7081

Recognizing that the purpose of breast cancer screening is to decrease breast cancer mortality and morbidity, NCCN continues to recommend annual screening mammography beginning at age 40 years for averagerisk women. Mammographic screening and subsequent treatment based on those results reduces breast cancer mortality based on a wide range of studies using various methodologies. ${ }^{1-4}$

Recent publicity emphasizing differences between various mammographic screening recommendations has masked fundamental areas of agreement among major organizations in the United States, all of which recommend routine screening mammography. Analysis by these key organizations, including the American Cancer Society (ACS) and US Preventive Services Task Force (USPSTF), have shown that the maximum mortality reduction and life years gained (LYG) benefit occurs when screening begins at age 40 years. ${ }^{1,2}$ All of the groups agree that screening mammography is an imperfect test with limitations, especially for women with dense breasts, and all advocate informed patient

aDepartment of Radiology and Rogel Cancer Center, University of Michigan, Ann Arbor, Michigan; and 'Department of Clinical Cancer Prevention, The University of Texas MD Anderson Cancer Center, Houston, Texas. Submitted June 7, 2018; accepted for publication August 21, 2018.

Dr. Helvie has disclosed that he has received grant/research support from GE Healthcare and IBM Watson. Dr. Bevers has disclosed that she has no decision-making regarding screening. Mammographic screening should not be offered to women with limited life expectancy.

This article highlights NCCN's position on screening mammography for average-risk women, emphasizing important factors considered by NCCN, but is not intended as a comprehensive screening review or assessment of emerging supplemental screening technologies covered elsewhere. ${ }^{5} \mathrm{NCCN}$ believes that women electing to undergo screening mammography should be counseled regarding potential benefits, risks, and limitations, and shared decision-making is encouraged.

Breast cancer is a major worldwide health problem. In the United States, $12.5 \%$, or 1 in 8 women, will develop breast cancer during their lifetime. In 2018, an estimated 266,120 cases of invasive breast cancer and 63,960 cases of in situ carcinoma will be diagnosed. ${ }^{6}$ In contrast, during the same year, 112,350 women will be diagnosed with lung cancer, 26,240 with pancreatic cancer, and 13,240 with cervical cancer. ${ }^{6}$ Breast cancer is the most common nonskin cancer impacting women:

financial interests, arrangements, affiliations, or commercial interests with the manufacturers of any products discussed in this article or their competitors.

Correspondence: Mark A. Helvie, MD, Department of Radiology, Rogel Cancer Center, University of Michigan, 2910 Taubman Center, SPC 5326 1500 East Medical Center Drive, Ann Arbor, MI 48109.

Email: mahelvie@med.umich.edu 
Screening Mammography for Average-Risk Women

an estimated 40,920 women will die of the disease in $2018 .^{6}$

Since 1990, the mortality rate in the United States has decreased by a remarkable 39\%, which has been attributed to advances in screening, treatment, and early detection. ${ }^{6}$ An even greater $49 \%$ mortality decline is estimated when adjustments are made for existing background mortality trends. ${ }^{7}$ Invasive breast cancer incidence in the United States has remained stable since the late 1980s. ${ }^{2}$ In contrast, WHO data shows worldwide mortality and incidence rates increased $(2.8 \%$ and $4 \%$ per year, respectively) from 2008 through $2012 .{ }^{8}$ The progress made in the United States and other countries is lacking in much of the world.

Why do organizations differ on screening recommendations? In substantial measure, these reveal different subjective value judgements between the benefits (deaths averted or LYG) versus the risks (harms). These differences also reflect whether the perspective is individual or population-based. Screening recommendations differ primarily in terms of age of initiation and frequency of screening, and these differences have caused confusion among women and providers regarding appropriate use of mammography, which may contribute to its current underuse. There has been more consistency regarding the age at which to stop screening and the need to integrate overall patient health in screening decisions.

Table 1 summarizes the guidelines by several major organizations. In 2016, in an attempt to harmonize the various recommendations, the American College of Obstetricians and Gynecologists (ACOG) held a consensus conference in Washington, DC, which included members from NCCN, American Academy of Family Physicians, ACS, American College of Physicians, American College of Radiology, American College of Surgeons, and USPSTF. The goal was to produce a screening document on which all organizations could agree. However, consensus could not be reached due to the differences regarding age of onset and frequency.

The NCCN Breast Cancer Screening and Diagnosis Panel subsequently convened in 2017 and affirmed that the primary purpose of screening is to decrease mortality and treatment-related morbidity. NCCN prioritized the benefits of screening over the known risks. In addition to mortality benefit,

\begin{tabular}{|c|c|c|c|}
\hline & Initiation Age & Frequency & Stopping Age \\
\hline $\mathrm{NCCN}^{5}$ & $40 y$ & Annual & $\begin{array}{l}\text { Consider severe } \\
\text { comorbidities } \\
\text { limiting life } \\
\text { expectancy }\end{array}$ \\
\hline $\mathrm{ACS}^{1}$ & $\begin{array}{l}40-44 \text { y: "Qualified"a } \\
45 \text { y: "Strong"a }\end{array}$ & $\begin{array}{l}\text { Annual: age } \\
40-54 \text { y } \\
\text { Biennial or } \\
\text { option annual: } \\
\text { age }>54 \text { y }\end{array}$ & $\begin{array}{l}\text { Life expectancy } \\
<10 \text { y }\end{array}$ \\
\hline USPSTF $^{2}$ & $\begin{array}{l}50 \text { y }\left(\text { grade } B^{b}\right) \\
40-49 \text { y }\left(\text { grade } C^{c}\right)\end{array}$ & Biennial & $\begin{array}{l}\text { Insufficient } \\
\text { evidence } \geq 75 \text { y }\end{array}$ \\
\hline
\end{tabular}

Abbreviations: ACS, American Cancer Society; USPSTF, US Preventive Services Task Force.

aSee text for ACS definitions of "qualified" and "strong" recommendations.

bUSPSTF recommends the service. There is high certainty that the net benefit is moderate or there is moderate certainty that the net benefit is moderate to substantial.

CUSPSTF recommends selectively offering or providing this service to individual patients based on professional judgment and patient preferences. There is at least moderate certainty that the net benefit is small.

mammography can confer morbidity benefit, including fewer mastectomies, fewer axillary dissections, and more limited use of chemotherapy compared with nonscreened women. ${ }^{9-12} \mathrm{NCCN}$ recognizes individual women will weigh benefits and risks differently, but believes women should have the opportunity to exercise their personal decision in choosing an appropriate screening strategy that allows maximal benefit, and that this should be a covered service.

A major impediment to organizational consensus is the inability to quantitate the value of a death averted or LYG compared with a nonlethal risk, such as a recall, needle breast biopsy, or potential overdiagnosed cancer. Women have placed a high value on the benefits of mortality reduction compared with the risks. Schwartz et $\mathrm{al}^{13}$ showed that $63 \%$ of women thought $\geq 500$ false-positives per life saved was acceptable, and $62 \%$ "did not want to take falsepositive results into account when deciding about screening."

Interestingly, since publication of the controversial 2009 USPSTF guidelines, $88 \%$ of surveyed internists, family medicine physicians, and gynecologists recommend screening mammography for women aged 45 to 49 years and $81 \%$ for women aged 40 to 44 years. ${ }^{14}$ These results show continued disconnect between certain organizational guidelines and practicing physicians' actions. 


\section{Evidence for Screening}

There are 2 major lines of evidence to support screening mammography: historic randomized controlled trials (RCTs) that assessed efficacy, and more recent observational population-based studies that have estimated effectiveness. Computer models have also been used to compare various screening regimens because direct $\mathrm{RCT}$ comparative evidence is lacking, but these models are subject to input assumptions. Most RCT studies used older film-screen mammography, which has been replaced by more effective digital mammography and, increasingly, digital tomosynthesis. The NCCN Breast Cancer Screening and Diagnosis Panel concluded that the evidence supports a category 1 recommendation for screening mammography to reduce breast cancer mortality ("Based upon high-level evidence, there is uniform NCCN consensus that the intervention is appropriate"). ${ }^{5}$

Combined analysis of RCTs, primarily conducted in Northern Europe, have shown a significant mortality reduction of approximately $20 \%$ among invited women aged 39 to 74 years. ${ }^{1,3}$ The benefit was not typically evident until 5 to 7 years after onset of screening. Too few women were aged $>74$ years to allow assessment. The RCTs were by invitation, not participation, and mimicked "intention to treat" and not "as treated," and hence they suffered from noncompliance and contamination. Mammography RCTs are now many decades old, with only one trial occurring in the United States that is 50 years old. Due to enormous changes in mammographic technique and changes in therapy, the application to current practice of these older studies is dated.

The recent ACS comprehensive screening review, using National Academy of Medicine methodology, emphasized a more contemporary analysis of case control and incidence-based mortality studies, which show greater benefit. ${ }^{1}$ Mortality reductions for case-control studies by invitation was $31 \%$ (odds ratio [OR], 0.69; 95\% CI, 0.57-0.83) and $48 \%$ (OR, 0.52; 95\% CI, 0.42-0.65) among attenders when corrected for self-selection. Among incidence-based studies, the reduction was 38\% (relative risk [RR], 0.62; 95\% CI, 0.56-0.69) among attenders. Although these studies lack the scientific rigor of RCTs, they reflect more current imaging and treatment methods. Contemporary North American studies have relevance to the US population.
Coldman et $\mathrm{al},{ }^{15}$ reporting on the Pan-Canadian screening experience involving 2.8 million women (aged 40-79 years), showed a 40\% (95\% CI, 33\%$48 \%$ ) mortality reduction among participating women across all age decades.

\section{Age to Begin Screening}

The NCCN Breast Cancer Screening and Diagnosis Panel affirmed age 40 years as the starting age based on mortality benefit for women aged 40 to 49 years. RCT meta-analysis showed an RR of 0.88 (CI, 0.73-1.0) for women aged 39 to 49 years. $^{2}$ Evidence for mortality reduction among women aged 40 to 49 years was judged as "high" by ACS. ${ }^{1}$ Among the 2 RCTs specific for ages 40 to 49 years, the UK Age trial showed a $25 \%$ mortality reduction (rate ratio, 0.75 ; 95\% CI, 0.58-0.97) after 10 years and a nonsignificant reduction (rate ratio, 0.88 ; 95\% CI, 0.74-1.04) after 17 years. ${ }^{16}$ The UK Age trial did not use digital mammography and limited imaging to one view, both of which are now known to decrease sensitivity. Modeling of the UK Age trial, assuming full compliance, demonstrated a $28 \%$ reduction (RR, 0.72; $95 \%$ CI, 0.65-0.75) at 13 years. ${ }^{17}$ The older Canadian National Breast Screening Study of women aged 40 to 49 years did not show benefit, ${ }^{18}$ but concerns have been expressed about its randomization techniques and image quality. More recent observational studies have shown mortality reduction for women aged 40 to 49 years. Hellquist et al, ${ }^{19}$ evaluating 7.3 million women-years of screening data in Sweden, showed a $29 \%$ mortality reduction (RR, 0.71; 95\% CI, 0.62 0.80 ) among attenders. Coldman et $\mathrm{al}^{15}$ showed a $44 \%$ reduction (standardized mortality ratio, 0.56 ; 95\% CI, 0.45-0.67) among Canadian women aged 40 to 49 years, a value similar to that observed for older women. The Cancer Intervention and Surveillance Modeling Network (CISNET) modeled benefit for annual screening at ages 40 to 49 years is $47 \mathrm{LYG} / 1,000$ women and 1.3 deaths averted/1,000 women. ${ }^{20}$ The same models estimate $122 \mathrm{LYG} / 1,000$ women for 25 years of biennial screening at ages 50 to 74 years, which equates to $49 \mathrm{LYG} / 1,000$ per decade, similar to the $47 \mathrm{LYG} / 1,000$ per decade benefit from annual screening at ages 40 to 49 years. ${ }^{20}$

Public confusion remains regarding the recent ACS and USPSTF recommendations for women aged 40 to 49 years. ACS recommends annual 
screening for women in their 40s; this is a "qualified recommendation" for women aged 40 to 44 years and a "strong recommendation" for those aged 45 to 49 years. ${ }^{1}$ From a patient perspective, ACS defined "qualified recommendation" as, "The majority of individuals in this situation would want the suggested course of action, but many would not. Patient preferences and informed decision making are desirable for making decisions." "Strong recommendation" was defined as, "Most individuals in this situation would want the recommended course of action and only a small proportion would not." ACS demonstrated substantial disease burden for women in their 40s, showing the most person-years of life lost to breast cancer for any 5-year period occurred at diagnosis in women aged 45 to 49 years. ${ }^{1}$ The 5 -year absolute breast cancer risk from age 45 to 49 years was $0.9 \%$, similar to the $1.1 \%$ at age 50 to 54 years. ${ }^{1}$ The USP. STF issued a grade $\mathrm{C}$ recommendation for biennial screening in women aged 40 to 49 years, and recommended an individual decision, but noted, "For women in their 40s, the benefit still outweighs the harms, but to a smaller degree." ${ }^{2}$ The NCCN recommendation is concordant with the combined strong and qualified recommendation issued by ACS for women in their 40s, but differs from that of the USPSTF.

\section{Screening Interval}

NCCN recommends annual screening, acknowledging the lack of RCT evidence on screening frequency. Given tumor growth patterns, more frequent screening would be expected to detect more clinically occult cancer. Assessment of interval cancer rates (palpable cancers occurring between screen dates) has shown higher rates when screening intervals increase. ${ }^{21-23}$ Retrospective studies suggest smaller or earlier-stage cancers are detected with annual versus biennial screening, although this advantage may decrease as women age. ${ }^{1,24,25}$ Computer models show more benefit with more frequent screening. ${ }^{17,26-28}$ For example, UK models for annual screening in women aged 40 to 73 years show a $36.7 \%$ mortality reduction, nearly double the $20.1 \%$ reduction for triennial screening. ${ }^{27}$ CISNET models of screening in women aged 40 to 74 years estimate $192 \mathrm{LYG/1,000}$ women for annual screening and 152 LYG/1,000 women for biennial screening, reflecting an improvement of $26 \%$ for annual screening. ${ }^{17}$ Compliance issues regarding annual versus biennial screening recommendations have been incompletely studied.

\section{Age to Stop Screening}

NCCN has not established an upper age for screening, but notes the decision should be based on severe comorbid conditions, which may limit life expectancy and a women's decision not to pursue treatment if diagnosed. Because RCTs show a lag in mortality benefit of 5 to 7 years, continuing screening among women with limited life expectancy does not seem warranted. The ACS has recommended 10 years of remaining life expectancy, which is the average life expectancy at age 80 years.

\section{Adverse Effects of Mammographic Screening}

All screening, including mammography, has the potential to subject women to adverse effects or risks (harms). Most women will not individually benefit from screening mammography (and most screening tests) because most will not develop breast cancer. NCCN is unaware of an accepted patient-centered metric that equates averting death from breast cancer with nonlethal risks. As such, the value assessment of risks is subjective and will vary among individuals. Restrictive screening recommendations that delay screening to age 50 years or advise less frequent intervals place a greater emphasis on the risks of screening, especially those related to false-positive results and overdiagnosis.

Frequently cited risks include recalls (or callbacks), false-positive biopsy results, and overdiagnosis. False-positives are often misunderstood, because recalls and false-positive biopsy results may be clumped together as "false-positives," although they reflect very different risks. A recall occurs when the original 2-view screening mammogram reflects a suspicious finding, which requires additional diagnostic mammograms or ultrasound to resolve. The federal Mammography Quality Standards Act considers these examinations "incomplete." ${ }^{29}$ Most recalls will be considered negative after additional imaging. False-positive biopsy results occur when a decision to recommend a biopsy leads to a benign 
pathologic result. The frequency of false-positive biopsy recommendations is $7.0 \%$ to $9.4 \%$ per decade of annual screening, depending on start age. ${ }^{2}$ Stated differently, an annually screened woman would expect to receive a false-positive biopsy recommendation averaging approximately once every 106 to 143 years of annual screening. Currently, most biopsies are performed as image-directed core needle biopsies in an outpatient setting using local anesthesia, which is the preferred biopsy method by NCCN. ${ }^{5}$ Overall, the estimated ratio of LYG benefit to falsepositive biopsy recommendation is 1.0 LYG per benign biopsy. ${ }^{30}$

Arguments to delay or restrict screening due to higher recall and false-positive biopsy rates in women in their 40s compared with those in their 50s have confused prevalent versus incidence screening considerations with starting age. There will be more recalls and false-positive biopsy recommendations at first (prevalent) screening, whether this occurs at age 40 or 50 years. Hubbard et $\mathrm{al}^{31}$ showed the per decade recall rate to be the same whether annual screening began at 40 or 50 years of age $(61 \%$ per decade or $6.1 \%$ per annual screening). The false-positive biopsy recommendation was higher when screening began at age 50 years ( $9.4 \%$ for 10 years) than at age 40 years ( $7.0 \%$ for 10 years). Annual screening from age 40 to 49 years results in slightly higher false-positive biopsy rates than biennial screening ( $7 \%$ vs $5 \%$ for 10 years, respectively). ${ }^{31}$ A proportion of false-positive biopsies, although currently classified as benign, reveal high-risk or premalignant conditions, such as lobular carcinoma in situ or atypical ductal hyperplasia, which allows for alternative risk management decisions and may be preventive. ${ }^{5}$

\section{Overdiagnosis}

Overdiagnosis is defined as breast cancer detected by screening that would not have become clinically apparent in a woman's lifetime by usual care. The main harm of overdiagnosis relates to its associated treatment. Most commonly, overdiagnosis is attributed to competing causes of death occurring during the lead time after screen detection. Overdiagnosis is primarily a consideration for older women who face more competing causes of death. For example, Hendrick ${ }^{32}$ calculated type 1 overdiagnosis to be $<1 \%$ for premenopausal women and $22 \%$ at age 80 years. There is an extremely wide range ( $-5 \%$ to $75 \%)$ of estimates of overdiagnosis related to screening reflecting a myriad of varying assumptions. ${ }^{1-3,33-41}$ All measurements have limitations, and prospective prediction of an overdiagnosed cancer at an individual level is not possible. The EUROSCREEN Working Group review estimated overdiagnosis (for combined invasive cancer and ductal carcinoma in situ) at $1 \%$ to $10 \%$ in their analysis of 16 studies after appropriate adjustments were made for lead time, risk, and background trends, factors glaringly absent in some studies. ${ }^{33}$ Overdiagnosis does not determine when to start screening or the interval at which to screen, but rather whether to screen. Neither the age at which to start screening nor the screen interval should substantially influence overdiagnosis, because these cancers would be expected to be persistent until screen-detected..$^{42}$ More fundamentally, the underlying premise of overdiagnosis is that the amount or level of diagnosis at usual clinical care is optimal or ideal and the screen level is excessive. However, the potential risk of overdiagnosis with screening must be balanced with the known harm of "overdying" without screening. Limiting the level of diagnosis to symptomatic disease is averse to many aspects of contemporary medicine, which seeks to avoid symptomatic disease by diagnosing and successfully treating presymptomatic conditions even as incidence increases. Hypertension is diagnosed and treated among asymptomatic individuals to avoid symptomatic detection at the time of a cardiovascular event, even though the incidence of screen-detected hypertension is much higher than symptomatic detection would be. Furthermore, the continuing advances in personalized treatment of newly diagnosed cancer will diminish the significance of overdiagnosis by reducing treatment morbidity.

NCCN believes the risks of screening should be balanced against the risk of not screening. The absence of mammographic screening does not equate with the absence of risk, as many assume. The net risks are the valid measure of risk. Nonscreened women frequently develop symptoms and undergo diagnostic physical examinations, mammographic imaging, and interventional biopsies, most with false-positive (benign) outcomes. Barton et $\mathrm{al}^{43}$ showed a $23 \%$ symptomatic presentation rate among women aged 40 to 69 years during a decade of observation (32\% among women aged $40-49$ years), with 
Screening Mammography for Average-Risk Women

$27 \%$ of symptomatic encounters leading to invasive procedures and an overall $6.2 \%$ positive predictive value. Importantly, there were significantly fewer symptomatic evaluations among screened women compared with nonscreened women. Symptomatic evaluations subject women to the risks of clinical encounters, anxiety, false-positive assessments, and potential for mammographic overdiagnosis.

But the greatest harm of not screening or screening less frequently is preventable death. Arleo et al, ${ }^{30}$ using CISNET model results, showed substantially higher benefit with annual screening from age 40 to 84 years compared with biennial screening from age 50 to 74 years. A $72 \%$ improvement in LYG (189 vs 110/1,000 women) was noted, with a similar improvement in mortality reduction (39.6\% vs $23.2 \%$ ). To achieve these improvements, more mammograms (36,550 vs $11,066 / 1,000$ women) and downstream testing would be performed. A total of 11.9 per 1,000 breast cancer deaths were estimated to be averted with annual screening beginning at 40 years. To put these numbers in perspective, 5.3 women need to be screened per each LYG when annual screening begins at 40 years. ${ }^{30}$

Because the purpose of screening is to decrease breast cancer mortality and morbidity, the NCCN Guidelines for Breast Cancer Screening and Diagnosis weigh the benefits substantially higher than the risks in determining the current recommendation of annual screening beginning at age 40 years. The panel recognizes that some women will choose to screen later, less frequently, or not at all. However, women should be provided with the opportunity to elect the screening regimen that has the highest associated benefit, which remains the purpose of screening.

\section{References}

1. Oeffinger KC, Fontham ET, Etzioni R, et al. Breast cancer screening for women at average risk: 2015 guideline update from the American Cancer Society. JAMA 2015;314:1599-1614.

2. Siu A; U.S. Preventive Services Task Force. Screening for breast cancer: U.S. Preventive Services Task Force recommendation statement. Ann Intern Med 2016;164:279-296.

3. Marmot MG, Altman DG, Cameron DA, et al. The benefits and harms of breast cancer screening: an independent review. Br J Cancer 2013;108:2205-2240.

4. Broeders M, Moss S, Nystrom L, et al. The impact of mammographic screening on breast cancer mortality in Europe: a review of observational studies. J Med Screen 2012;19(Suppl 1):14-25.

5. Bevers TB, Helvie MA, Bonaccio E, et al. NCCN Clinical Practice Guidelines in Oncology: Breast Cancer Screening and Diagnosis. Version 3.2018. Accessed October 2, 2018. To view the most recent version of these guidelines, visit NCCN.org.

6. Siegel RL, Miller KD, Jemal A. Cancer statistics, 2018. CA Cancer J Clin 2018;68:7-30.

7. Plevritis SK, Munoz D, Kurian AW, et al. Association of screening and treatment with breast cancer mortality by molecular subtype in US women, 2000-2012. JAMA 2018;319:154-164.

8. World Health Organization/International Agency for Research on Cancer Latest World Cancer Statistics. Global Cancer Burden Rises to 14.1 Million New Cases in 2012: Marked Increase in Breast Cancers Must Be Addressed. Available at: https://www.iarc.fr/en/media-centre/pr/2013/pdfs/ pr223_E.pdf. Accessed October 2, 2018.

9. Malmgren JA, Parikh J, Atwood MK, Kaplan HG. Impact of mammography detection on the course of breast cancer in women aged 40-49 years. Radiology 2012;262:797-806.

10. Coldman AJ, Phillips N, Speers C. A retrospective study of the effect of participation in screening mammography on the use of chemotherapy and breast conserving surgery. Int J Cancer 2007;120:2185-2190.

11. Zorzi M, Puliti D, Vettorazzi $M$, et al. Mastectomy rates are decreasing in the era of service screening: a population-based study in Italy (1997-2001). Br J Cancer 2006;95:1265-1268.

12. Plecha D, Salem N, Kremer $M$, et al. Neglecting to screen women between 40 and 49 years old with mammography: what is the impact on treatment morbidity and potential risk reduction? AJR Am J Roentgenol 2014;202:282-288.

13. Schwartz LM, Woloshin S, Sox HC, et al. US women's attitudes to false positive mammography results and detection of ductal carcinoma in situ: cross sectional survey. BMJ 2000;320:1635-1640.
14. Radhakrishnan A, Nowak SA, Parker AM, et al. Physician breast cancer screening recommendations following guideline changes: results of a national survey. JAMA Intern Med 2017;177:877-878.

15. Coldman A, Phillips N, Wilson C, et al. Pan-Canadian study of mammography screening and mortality from breast cancer. J Natl Cancer Inst 2015;107:dju404.

16. Moss SM, Wale C, Smith R, et al. Effect of mammographic screening from age 40 years on breast cancer mortality in the UK Age trial at 17 years' follow-up: a randomised controlled trial. Lancet Oncol 2015;16:11231132 .

17. Mandelblatt JS, Stout NK, Schechter CB, et al. Collaborative modeling of the benefits and harms associated with different U.S. breast cancer screening strategies. Ann Intern Med 2016;164:215-225.

18. Miller AB, Wall C, Baines CJ, et al. Twenty five year follow-up for breast cancer incidence and mortality of the Canadian National Breast Screening Study: randomised screening trial. BMJ 2014;348:g366.

19. Hellquist BN, Duffy SW, Abdsaleh S, et al. Effectiveness of populationbased service screening with mammography for women ages 40 to 49 years: evaluation of the Swedish Mammography Screening in Young Women (SCRY) cohort. Cancer 2011;117:714-722.

20. Mandelblatt J, Cronin K, de Koning H, et al. Technical Report: Collaborative Modeling of U.S. Breast Cancer Screening Strategies. Available at: file://C:/Users/green/Downloads/collabmodelingbc.pdf. Accessed October 2, 2018

21. Bennett RL, Sellars SJ, Moss SM. Interval cancers in the NHS breast cancer screening programme in England, Wales and Northern Ireland. $\mathrm{Br}$ J Cancer 2011;104:571-577.

22. Winch CJ, Sherman KA, Boyages J. Toward the breast screening balance sheet: cumulative risk of false positives for annual versus biennial mammograms commencing at age 40 or 50 . Breast Cancer Res Treat 2015;149:211-221.

23. Tabar L, Fagerberg G, Chen HH, et al. Efficacy of breast cancer screening by age. New results from the Swedish two-county trial. Cancer 1995;75:25072517.

24. Miglioretti DL, Zhu W, Kerlikowske K, et al. Breast tumor prognostic characteristics and biennial vs annual mammography, age, and menopausal status. JAMA Oncol 2015;1:1069-1077.

25. Hunt KA, Rosen EL, Sickles EA. Outcome analysis for women undergoing annual versus biennial screening mammography: a review of 24,211 examinations. AJR Am J Roentgenol 1999;173:285-289.

26. Michaelson J, Halpern E, Kopans D. Breast cancer: computer simulation method for estimating optimal intervals for screening. Radiology $1999 ; 212: 551-560$. 
27. Gunsoy NB, Garcia-Closas M, Moss SM. Estimating breast cancer mortality reduction and overdiagnosis due to screening for different strategies in the United Kingdom. Br J Cancer 2014;110:2412-2419.

28. Mandelblatt JS, Cronin KA, Bailey S, et al. Effects of mammography screening under different screening schedules: model estimates of potential benefits and harms. Ann Intern Med 2009;151:738-747.

29. Quality mammography standards-FDA. Final rule. Fed Regist 1997;62:55852-55994.

30. Arleo EK, Hendrick RE, Helvie MA, Sickles EA. Comparison of recommendations for screening mammography using CISNET models. Cancer 2017;123:3673-3680.

31. Hubbard RA, Kerlikowske K, Flowers CI, et al. Cumulative probability of false-positive recall or biopsy recommendation after 10 years of screening mammography: a cohort study. Ann Intern Med 2011;155:481-492.

32. Hendrick RE. Obligate overdiagnosis due to mammographic screening: a direct estimate for U.S. women. Radiology 2018;287:391-397.

33. Puliti D, Duffy SW, Miccinesi G, et al. Overdiagnosis in mammographic screening for breast cancer in Europe: a literature review. J Med Screen 2012;19(Suppl 1):42-56.

34. Bleyer A, Welch HG. Effect of three decades of screening mammography on breast-cancer incidence. N Engl J Med 2012;367:1998-2005.

35. Zackrisson S, Andersson I, Janzon L, et al. Rate of over-diagnosis of breast cancer 15 years after end of Malmo mammographic screening trial: followup study. BMJ 2006;332:689-692.
36. Njor SH, Olsen AH, Blichert-Toft M, et al. Overdiagnosis in screening mammography in Denmark: population based cohort study. BM] 2013;346:f1064.

37. Hellquist BN, Duffy SW, Nystrom L, Jonsson H. Overdiagnosis in the population-based service screening programme with mammography for women aged 40 to 49 years in Sweden. J Med Screen 2012;19:14-19.

38. Duffy SW, Dibden A, Michalopoulos D, et al. Screen detection of ductal carcinoma in situ and subsequent incidence of invasive interval breast cancers: a retrospective population-based study. Lancet Oncol 2016;17:109-114

39. Johns LE, Coleman DA, Swerdlow AJ, Moss SM. Effect of population breast screening on breast cancer mortality up to 2005 in England and Wales: an individual-level cohort study. Br J Cancer 2016;116:246-252.

40. Lauby-Secretan B, Scoccianti C, Loomis D, et al. Breast-cancer screening-viewpoint of the IARC Working Group. N Engl J Med 2015;372:2353-2358.

41. Helvie MA, Chang JT, Hendrick RE, Banerjee M. Reduction in latestage breast cancer incidence in the mammography era: implications for overdiagnosis of invasive cancer. Cancer 2014;120:2649-2656.

42. Arleo EK, Monticciolo DL, Monsees B, et al. Persistent untreated screening-detected breast cancer: an argument against delaying screening or increasing the interval between screenings. J Am Coll Radiol 2017;14:863-867

43. Barton MB, Elmore JG, Fletcher SW. Breast symptoms among women enrolled in a health maintenance organization: frequency, evaluation, and outcome. Ann Intern Med 1999;130:651-657. 